\title{
Fecal microbiota transplantation for recurrent Clostridium difficile infection in a patient with ulcerative colitis
}

\author{
Kosaku Nanki ${ }^{1}$, Shinta Mizuno ${ }^{1}$, Katsuyoshi Matsuoka ${ }^{2}$, Keiko Ono ${ }^{1}$, Shinya Sugimoto ${ }^{1}$, Hiroki Kiyohara ${ }^{1}$, \\ Mari Arai ${ }^{1}$, Moeko Nakashima ${ }^{1}$, Kozue Takeshita ${ }^{1}$, Keiichiro Saigusa ${ }^{1}$, Mitsutoshi Senoh ${ }^{3}$, Tadashi Fukuda ${ }^{3}$, \\ Makoto Naganuma ${ }^{1}$, Haru Kato ${ }^{3}$, Wataru Suda ${ }^{4}$, Masahira Hattori ${ }^{5}$, Takanori Kanai ${ }^{1}$ \\ ${ }^{1}$ Division of Gastroenterology and Hepatology, Department of Internal Medicine, Keio University School of Medicine, Tokyo, ${ }^{2}$ Department of \\ Gastroenterology and Hepatology, Tokyo Medical and Dental University, Tokyo, ${ }^{3}$ Department of Bacteriology II, National Institute of Infectious \\ Diseases, Tokyo, ${ }^{4}$ Graduate School of Frontier Sciences, University of Tokyo, Chiba, ${ }^{5}$ Faculty of Science and Engineering, Waseda University, \\ Tokyo, Japan
}

Fecal microbiota transplantation (FMT) has been reported as a safe and effective therapy in patients with refractory and recurrent Clostridium difficile infection (CDI). FMT has also been reported as a promising therapy in patients with ulcerative colitis (UC). Both, CDI and UC, are believed to be caused by dysbiosis, such as altered compositions or decreased diversity of the intestinal microbiota. This report describes a patient with UC in remission with a second recurrent episode of CDI, who was treated with FMT. A single FMT performed via colonoscopy completely resolved the patient's diarrhea and eradicated $C$. difficile bacteriologically without any severe complications. Molecular biological analysis of the patient's fecal microbiota showed that FMT could dramatically change the altered composition of intestinal microbiota and restore its diversity. Despite the restoration of the intestinal microbiota, FMT could not prevent a relapse of UC in this patient. However, it improved the intestinal symptoms of CDI and could prevent further recurrences of CDI. (Intest Res 2018;16:142-146)

Key Words: Fecal microbiota transplantation; Clostridium difficile; Colitis, ulcerative

\section{INTRODUCTION}

Clostridium difficile is a gram-positive, spore-forming bacterium, which causes antibiotic-associated colitis. UC is a risk factor for $C$. difficile infection (CDI). ${ }^{1}$ Although CDI is widely treated with antibiotics such as metronidazole and vancomycin, it has a high recurrence rate. Fecal microbiota transplantation (FMT) has been shown to be an effective and safe alternative therapy in patients with multiple recurrences of CDI. ${ }^{2}$ FMT can not only treat CDI but also normalize the imbalanced gut microbiota, a condition called dys-

Received February 6, 2017. Revised March 28, 2017.

Accepted April 5, 2017. Published online August 31, 2017

Correspondence to Takanori Kanai, Division of Gastroenterology and

Hepatology, Department of Internal Medicine, Keio University School of

Medicine, 35 Shinanomachi, Shinjuku-ku, Tokyo 160-8582, Japan.

Tel: +81-3-3353-1211, Fax: +81-3-3341-3631,E-mail: takagast@keio.jp biosis. ${ }^{3}$ The guidelines of the American Gastroenterological Association and the European Society of Clinical Microbiology and Infectious Diseases highly recommend FMT for patients who experience multiple recurrences of CDI despite treatment with currently available antibiotics. ${ }^{4,5}$

Although the incidence of recurrent and refractory CDI has increased significantly worldwide, ${ }^{6}$ only 1 case report to date has reported the effects of FMT in patients with CDI in Japan. ${ }^{7}$ Furthermore, FMT may be a promising treatment in patients with UC as well. We recently conducted a clinical trial assessing the efficacy and safety of FMT in patients with UC, CDI, and intestinal Behçet's disease (UMIN registration No. 000012814; http://www.umin.ac.jp/). ${ }^{8}$ Although several reports have described the use of FMT in patients with UC with concurrent CDI, only one has described the clinical onset and analysis of the changes in the gut's microbiota. ${ }^{9}$ Here, we describe a case of successful FMT in a patient with UC

\footnotetext{
๑ Copyright 2018. Korean Association for the Study of Intestinal Diseases. All rights reserved.

This is an Open Access article distributed under the terms of the Creative Commons Attribution Non-Commercial License (http://creativecommons.org/licenses/by-nc/4.0)

which permits unrestricted non-commercial use, distribution, and reproduction in any medium, provided the original work is properly cited.
} 
and recurrent CDI, as well as an analysis of the microbiota of fecal samples obtained before and after FMT.

\section{CASE REPORT}

An 82-year-old woman was referred to the Department of Gastroenterology at Keio University Hospital for unresolved diarrhea. Five months earlier, she had been diagnosed with left-sided mild active UC and treated with oral 5-aminosalicylic acid (5-ASA), which resolved the diarrhea. During the treatment of UC, she was administered 2 weeks of antibiotics for fever and suspected bacterial infection. Two months after the antibiotic treatment, she presented at another hospital with watery diarrhea, loss of appetite and was admitted for further evaluation. Stool examination by enzyme immunoassay (EIA) revealed toxins A and B. She was administered 2 courses of oral vancomycin (500 mg 4 times per day), which resolved her diarrhea and loss of appetite. However, CDI recurred a few days after each treatment. Therefore, she was referred to our hospital to undergo FMT, with her 48-yearold daughter as the donor. After signing the informed consent, she participated in a clinical trial of FMT approved by the ethics committees of Keio University on February 24, 2014.

Donor's stool was screened for transmissible diseases and pathogens, including $C$. difficile. Donor's blood was tested for HAV, HBV, HCV, human immunodeficiency, and human Tcell lymphotropic type 1 viruses, and Treponema pallidum. All these test results were negative. The donor had no history of IBS, IBD, diabetes mellitus, chronic diarrhea, chronic constipation, atopic dermatitis, morbid obesity, or cancer. On the morning of transplantation, fresh donor feces were collected. One hundred grams of the donated fecal matter were mixed with $200 \mathrm{~mL}$ of normal saline and homogenized. The fecal suspension was filtered with a sterile coarse metallic mesh, fine metallic mesh, and cotton gauze to remove large masses of feces and smoothen the texture. Following bowel lavage with $2 \mathrm{~L}$ of polyethylene glycol, the recipient underwent FMT via colonoscopy. The colonoscopy revealed diffuse erythematous mucosa with edema throughout the entire colon (Fig. 1).

On post-FMT day 4 , the symptoms of diarrhea, fecal incontinence, and abdominal distension resolved without any complications. EIA test was negative for fecal toxins,
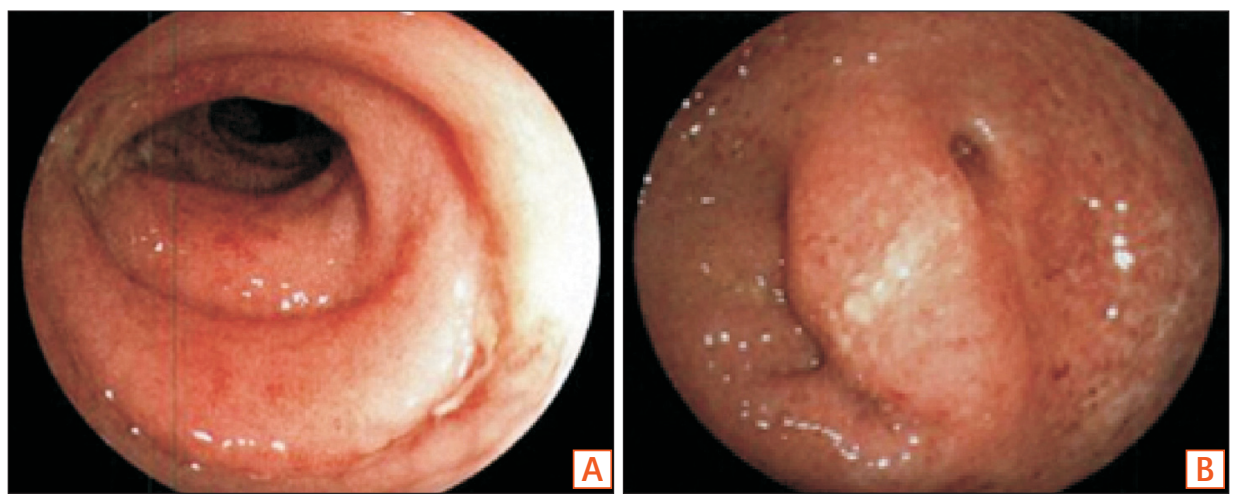

Fig. 1. Colonoscopic images before fecal microbiota transplantation. (A) Descending colon and (B) sigmoid colon.

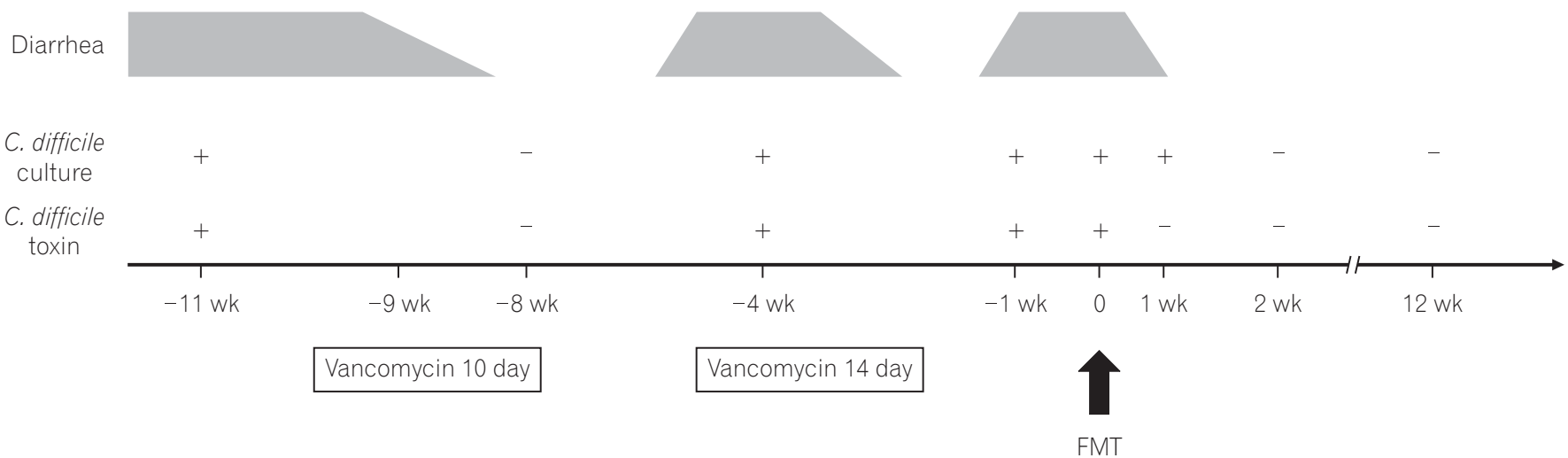

Fig. 2. The clinical course of the patient. C. difficile, Clostridium difficile; FMT, fecal microbiota transplantation. 
although stool culture yielded toxigenic $C$. difficile. On postFMT day 11, her stool culture was negative for $C$. difficile (Fig. 2).

C. difficile isolates from the first and third CDI episodes were available for analysis. Both isolates were positive for toxins $\mathrm{A}$ and $\mathrm{B}$, but negative for binary toxin (actin specific ADP-ribosyltransferase). ${ }^{10}$ PCR-ribotype analysis showed that both isolates were ribotype 014 (Fig. 3). The gut microbi-

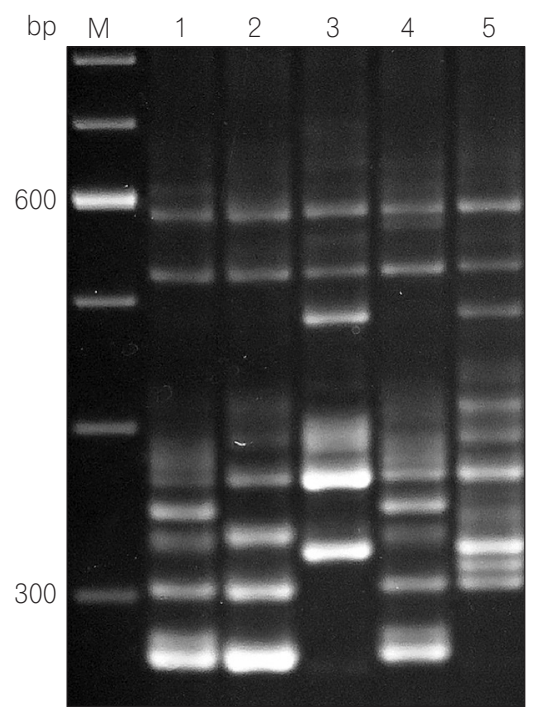

Fig. 3. PCR ribotyping patterns of Clostridium difficile isolated from the present patient and 4 ribotype strains (toxin A-positive, toxin B-positive, and binary toxin-negative) endemic in Japan. Lane $M_{1}$ molecular size marker (100 bp ladder); lane 1, C. difficile isolated from the present patient; lane 2, PCR-ribotype 001; lane 3, PCR-ribotype 002; lane 4, PCRribotype 014; lane 5, PCR-ribotype 018.

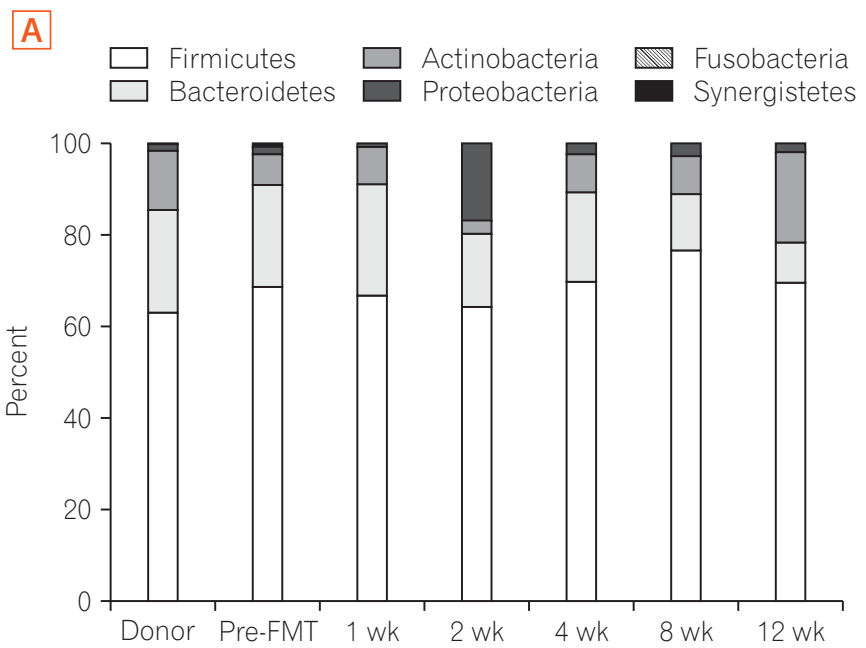

ota of the stool specimens obtained from the patient before FMT and 1, 2, 4, 8, and 12 weeks after FMT were analyzed by pyrosequencing of the bacterial $16 \mathrm{~S}$ rRNA gene (Fig. 4A). ${ }^{11}$ Operational taxonomic unit (OTU) analysis of $C$. difficile showed that the stool sample before FMT was positive for $C$. difficile OTU, but the stool samples after FMT were negative. Furthermore, the total number of OTUs before FMT was under 100, whereas the total number of OTUs after FMT was 150 to 200. Shannon index improved from 2.8 to about 3.5 after FMT (Fig. 4B). These results indicated that FMT could restore the diversity of the microbiota in the intestines and induce symbiosis.

No recurrence of CDI was observed for 1 year after FMT. UC remained in remission, as observed in clinical symptoms and endoscopic findings, before and until 5 months after FMT. Five months after FMT, the patient presented with stools containing mucous and blood. Testing for toxins A/B by EIA was negative. We diagnosed the patient with UC relapse without CDI recurrence and her partial Mayo (pMayo) score was 4 . Additional suppository treatment with 5-ASA was needed. Five weeks of treatment with topical 5-ASA resulted in remission and reduced the pMayo score to 1 .

\section{DISCUSSION}

CDI is considered causally related to disrupted colonization resistance after the administration of specific medicines, including antibiotics. These agents reduce the diversity or disrupt the composition of intestinal microbiota. FMT is believed to treat recurrent CDI with high efficacy by restor-

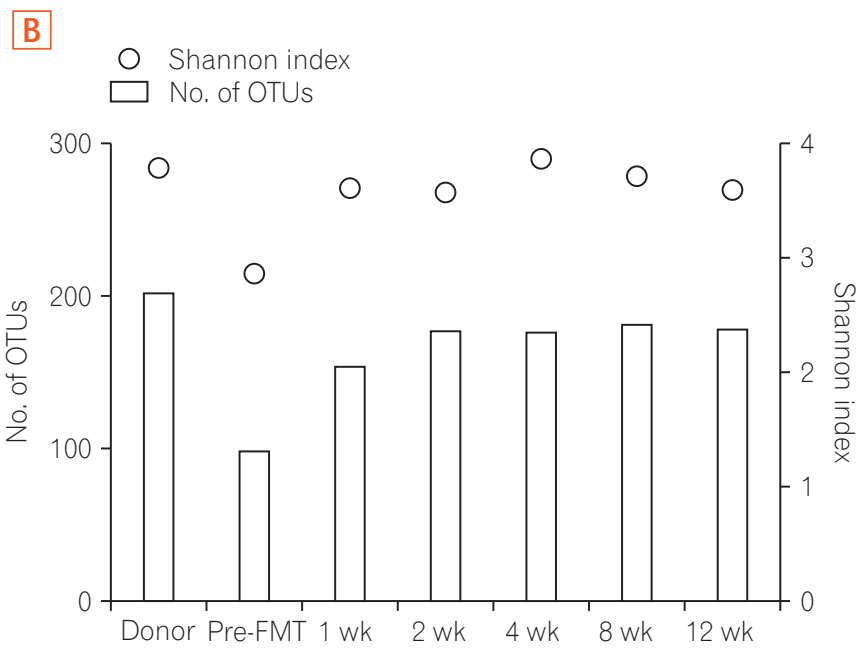

Fig. 4. Composition and diversity of the gut microbiome. (A) Phylum level taxonomic profiles of the donor and recipient, before and at 1, 2, 4, 8, and 12 weeks after fecal microbiota transplantation (FMT). (B) Microbiota diversity (Shannon index, circle) and total number of operational taxonomic unit (OTUs, bar) of the donor and recipient, before and at 1, 2, 4, 8, and 12 weeks after FMT. 
ing the diversity of intestinal microbiota and re-establishing colonization resistance..$^{12}$ An analysis of fecal microbiota before and after FMT showed that the diversity of microbiota, which was reduced before FMT, was restored after FMT. ${ }^{2}$ In the present case, FMT also dramatically restored the diversity of the gut microbiota.

The clinical guidelines of the Infection Diseases Society of America (IDSA) and the Society for Healthcare Epidemiology of America (SHEA) have classified the severity of CDI from mild to severe, with the latter including a peak white blood cell count $>15,000 / \mu \mathrm{L}$ and a peak serum creatinine level $>1.5$ times the premorbid level. ${ }^{13}$ These guidelines also recommend that the initial treatment should be based on the disease severity. The current patient had moderately severe CDI. A recent review of the management of CDI in IBD recommended the use of vancomycin but not metronidazole in patients with CDI and IBD, including UC. This is because IBD can be considered as another marker of the severity of CDI that indicates the need for more aggressive management with vancomycin or FMT..$^{14}$ Although IDSA and SHEA guidelines recommend oral metronidazole in patients with moderate severity of CDI, this patient was initially treated with oral vancomycin due to her comorbidity of UC, in accordance with the recent review of management of CDI in IBD. Almost $90 \%$ of the patients with CDI show improvements after the initial treatment, but $10 \%$ to $30 \%$ of these patients experience recurrent CDI within 8 weeks after the completion of the initial treatment. Factors associated with a high risk of recurrence include older age ( $>65$ years), continued use of non-CDI antibiotics after the diagnosis and/or treatment of CDI, comorbidities, including severe underlying disease and/or renal failure, a history of previous CDI (more than 1 recurrence), concomitant use of proton pump inhibitors, and initially severe disease. ${ }^{5}$ This patient had 2 risk factors for recurrence, older age and concomitant use of proton pump inhibitors, and she was at a moderate risk for CDI recurrence. Additionally, concurrent UC in this patient may have been a risk factor for CDI recurrence. Since comorbidity of UC is considered a risk factor for CDI recurrence, this patient required FMT as an aggressive therapy for CDI. Furthermore, concurrent CDI is a factor that can exacerbate UC. ${ }^{14}$ Upon discussion of the risks and benefits of FMT and antibiotic therapy such as vancomycin for recurrent CDI in patients with UC, FMT was considered a suitable treatment for the third episode of CDI in the present patient.

Typical colonoscopic findings of CDI, such as pseudomembranes, are often absent in patients with UC. ${ }^{15}$ Colonoscopic examination of this patient showed no evidence of pseudomembranous colitis. Symptoms, such as diarrhea, are similar in patients with UC and CDI. Patients with UC who experience flare-ups should be assessed for CDI, even if their colonoscopy findings do not reveal pseudomembranous colitis.

Many methods have been used to administer donated fecal materials, including a nasogastric or nasojejunal tube, esophagogastroduodenoscopy, retention enema, and colonoscopy. A meta-analysis in patients with CDI showed that FMT was more effective when delivered through the lower than the upper gastrointestinal tract; however, no individual study has directly compared these delivery methods. ${ }^{16} \mathrm{We}$ chose to administer FMT via colonoscopy because administration through the upper gastrointestinal tract carries risks of vomiting and aspiration in older patients, whereas colonoscopy enables the inoculation of fecal material throughout the entire colon. Well-designed randomized controlled trials are needed to determine the methodologies that are most effective and safe for patients with recurrent CDI.

Our patient also had a history of UC, which became exacerbated 5 months after FMT. The pathophysiology of UC is thought to be related to imbalances in intestinal microbiota. ${ }^{17}$ It has been reported that phylum Firmicutes and Bacteroidetes are decreased in the patients with UC than in healthy subjects. However, in the present case, the proportions of phylum Firmicutes and Bacteroidetes in the gut microbiota of the patient were similar to those of the donor. These findings were considered the result of her remission state of UC. Studies in pathological animal models have suggested that altered microbiota could induce the development of a UC-like colitis. ${ }^{18}$ However, the efficacy of FMT in patients with UC remains unclear. ${ }^{19,20}$ FMT was not considered harmful in this patient, because UC did not flare up within at least 4 months after FMT. Our finding, that a single FMT cured recurrent CDI, suggests that FMT could induce bacterial symbiosis in the gut. However, despite this symbiosis, our patient experienced a UC flare-up 5 months later. These findings suggest that FMT was ineffective in UC, or a single FMT was not sufficient to maintain the remission of UC. Furthermore, well-designed randomized controlled trials are needed to confirm the efficacy of FMT in patients with UC.

In summary, we found that a single FMT completely cured recurrent CDI in a patient with concurrent UC. However, FMT did not sustain remission of UC, suggesting that FMT has different efficacies in CDI and UC. 


\section{FINANCIAL SUPPORT}

This study was supported in part by Health and Labour Sciences Research Grants for research on intractable diseases from the Japanese Ministry of Health.

\section{CONFLICT OF INTEREST}

No potential conflict of interest relevant to this article was reported.

\section{AUTHOR CONTRIBUTION}

K.N., S.M., K.O., S.S., H.K., M.A., K.T., and K.S. performed fecal microbiota transplantation. Moeko Nakashima, W.S., and M.H. performed analysis of the stool specimens. M.S., T.F., and H.K. performed PCR-ribotype analysis. K.M., Makoto Naganuma and T.K. conceived and conducted the clinical trial of FMT. K.N. and S.M. wrote the manuscript.

\section{ACKNOWLEDGEMENTS}

The authors thank K. Mori, M. Mutaguchi, Y. Nakazato, R. Bessho, N. Inoue, Y. Iwao, and H. Ogata of Keio University for useful discussions.

\section{REFERENCES}

1. Sinh P, Barrett TA, Yun L. Clostridium difficile infection and inflammatory bowel disease: a review. Gastroenterol Res Pract 2011;2011:136064.

2. van Nood E, Vrieze A, Nieuwdorp M, et al. Duodenal infusion of donor feces for recurrent Clostridium difficile. N Engl J Med 2013;368:407-415.

3. Brandt LJ. American Journal of Gastroenterology Lecture: intestinal microbiota and the role of fecal microbiota transplant (FMT) in treatment of C. difficile infection. Am J Gastroenterol 2013;108:177-185.

4. Surawicz CM, Brandt LJ, Binion DG, et al. Guidelines for diagnosis, treatment, and prevention of Clostridium difficile infections. Am J Gastroenterol 2013;108:478-498.

5. Debast SB, Bauer MP, Kuijper EJ; European Society of Clinical Microbiology and Infectious Diseases. European society of clinical microbiology and infectious diseases: update of the treatment guidance document for Clostridium difficile infection. Clin Microbiol Infect 2014;20 Suppl 2:1-26.

6. Lessa FC, Mu Y, Bamberg WM, et al. Burden of Clostridium difficile infection in the United States. N Engl J Med 2015;372:825834.
7. Tanaka T, Kato H, Fujimoto T. Successful fecal microbiota transplantation as an initial therapy for Clostridium difficile infection on an outpatient basis. Intern Med 2016;55:999-1000.

8. Matsuoka K, Mizuno S, Hayashi A, Hisamatsu T, Naganuma M, Kanai T. Fecal microbiota transplantation for gastrointestinal diseases. Keio J Med 2014;63:69-74.

9. Brace C, Gloor GB, Ropeleski M, Allen-Vercoe E, Petrof EO. Microbial composition analysis of Clostridium difficile infections in an ulcerative colitis patient treated with multiple fecal microbiota transplantations. J Crohns Colitis 2014;8:1133-1137.

10. Kato $\mathrm{H}$, Kato $\mathrm{N}$, Watanabe $\mathrm{K}$, et al. Identification of toxin Anegative, toxin B-positive Clostridium difficile by PCR. J Clin Microbiol 1998;36:2178-2182.

11. Kim SW, Suda W, Kim S, et al. Robustness of gut microbiota of healthy adults in response to probiotic intervention revealed by high-throughput pyrosequencing. DNA Res 2013;20:241-253.

12. Lawley TD, Clare S, Walker AW, et al. Targeted restoration of the intestinal microbiota with a simple, defined bacteriotherapy resolves relapsing Clostridium difficile disease in mice. PLoS Pathog 2012;8:e1002995. doi: 10.1371/journal.ppat.1002995.

13. Mulherin DW, Hutchison AM, Thomas GJ, Hansen RA, Childress DT. Concordance of the SHEA-IDSA severity classification for Clostridium difficile infection and the ATLAS bedside scoring system in hospitalized adult patients. Infection 2014;42:999-1005.

14. Khanna S, Shin A, Kelly CP. Management of Clostridium difficile infection in inflammatory bowel disease: expert review from the clinical practice updates committee of the AGA institute. Clin Gastroenterol Hepatol 2017;15:166-174.

15. Issa M, Vijayapal A, Graham MB, et al. Impact of Clostridium difficile on inflammatory bowel disease. Clin Gastroenterol Hepatol 2007;5:345-351.

16. Kassam Z, Lee CH, Yuan Y, Hunt RH. Fecal microbiota transplantation for Clostridium difficile infection: systematic review and meta-analysis. Am J Gastroenterol 2013;108:500-508.

17. Frank DN, St Amand AL, Feldman RA, Boedeker EC, Harpaz N, Pace NR. Molecular-phylogenetic characterization of microbial community imbalances in human inflammatory bowel diseases. Proc Natl Acad Sci U S A 2007;104:13780-13785.

18. Garrett WS, Lord GM, Punit S, et al. Communicable ulcerative colitis induced by T-bet deficiency in the innate immune system. Cell 2007;131:33-45.

19. Rossen NG, Fuentes S, van der Spek MJ, et al. Findings from a randomized controlled trial of fecal transplantation for patients with ulcerative colitis. Gastroenterology 2015;149:110-118.e4.

20. Moayyedi P, Surette MG, Kim PT, et al. Fecal microbiota transplantation induces remission in patients with active ulcerative colitis in a randomized controlled trial. Gastroenterology 2015;149:102-109.e6. 